\title{
The Activation of Protein Kinase A Pathway Selectively Inhibits Anterograde Axonal Transport of Vesicles but Not Mitochondria Transport or Retrograde Transport in vivo
}

\author{
Yasushi Okada, Reiko Sato-Yoshitake, and Nobutaka Hirokawa \\ Department of Anatomy and Cell Biology, Faculty of Medicine, University of Tokyo, Hongo, Tokyo, 113, Japan
}

To shed light on how axonal transport is regulated, we examined the possible roles of protein kinase A (PKA) in vivo suggested by our previous work (Sato-Yoshitake et al., 1992). Pharmacological probes or the purified catalytic subunit of PKA were applied to the permeabilized-reactivated model of crayfish walking leg giant axon, and the effect was monitored by the quantitative video-enhanced light microscopy and the quantitative electron microscopy. Dibutyryl cyclic AMP caused concentration-dependent transient reduction in the number of anterogradely transported small vesicles, while the retrogradely transported organelles and anterogradely transported mitochondria showed no decrease. This transient selective inhibition of anterograde vesicle transport was reversed by the application of a specific inhibitor of PKA (KT5720) in a concentration-dependent manner, and was reproduced by the application of the purified catalytic subunit of PKA and augmented by the application of adenosine 5'-0-(3-thiotriphosphate) (ATP $\gamma \mathrm{S}$ ). Corresponding biochemical study showed that several axoplasmic proteins including kinesin were specifically phosphorylated by the activation of the PKA pathway. These findings suggest the possible roles of PKA in the regulation of the direction of the axonal transport in vivo. The finding that only vesicle transport but not mitochondria transport was inhibited also suggests that the transport of vesicles and that of mitochondria are differently regulated and might be supported by different motors.

[Key words: axonal transport, phosphorylation, kinesin, protein kinase A, phosphatase, crayfish walking leg giant axon]

Numerous types of membranc-bound organclles are transported and distributed through the cytoplasm. Fast axonal transport, a bidirectional transport of membranous organelles through axon,

\footnotetext{
Received Sept. 12, 1994; revised Nov. 2, 1994; accepted Nov. 8, 1994.

We thank Dr. Masaki Inagaki (Tokyo Metropolitan Institute of Gerontology) for the generous gift of the catalytic subunit of PKA, Dr. G. S. Bloom (University of Texas) for providing anti-kinesin antibody (H2), and Dr. N. Lomax (the National Cancer Institute) for supplying us with taxol. We also thank Dr Y. Ihara (Department of Neuropathology, University of Tokyo) for technical comments on phosphorylation experiments, and the members of our laboratory, especially Drs. H. Aizawa, T. Nakata, S. Okabe, and H. Yorifuji, for their invaluable advice and discussions throughout. This work was supported by a Special Grant-in-Aid for Scientific Research from the Japan Ministry of Education, Science and Culture, and by a grant fion the Institute of Physical and Chemical Research (RIKEN) to N.H. Y.O. was supported by a JSPS fellowship for Japanese Junior Scientists.

Correspondence should be addressed to N. Hirokawa at the above address.

Copyright $(1995$ Society for Neuroscience $0270-6474 / 95 / 153053-12 \$ 05.00 / 0$
}

is an especially well developed intracellular organelle transport system. Many studies have been focused on the mechanism of this transport, but little is known about its regulation. As a putative mechanism for the regulation, we have proposed a model from our recent studies (Hirokawa et al., 1990, 1991) that the anterograde motor kinesin is decommissioned at the axon terminal, and that the retrograde motor cytoplasmic dynein is transported, presumably in an inactive form, by the anterograde organelle transport to the axon terminal, where it should be activated to support the retrograde organelle transport.

Our present study was undertaken to examine this regulation mechanism. One plausible mechanism is through protein kinases and phosphatases, as is true with fish scale chromophores. In these cells, pigment granule dispersion and aggregation along microtubules are regulated through the protein kinase A (PKA) system (Lynch et al., 1986a,b; Rodzial and Haimo, 1986a,b; Sammak et al., 1992). It is not unreasonable to suppose that it might regulate the direction of the fast axonal transport as well, because PKA is reported to be enriched in brain, especially at the presynaptic terminal (Walter et al., 1978; Dunkley et al., 1988). This speculation was supported by our previous investigation (Sato-Yoshitake et al., 1992), in which we demonstrated that kinesin was phosphorylated by PKA, and that the phosphorylation reduced the affinity of kinesin to purified synaptic vesicles, while its motor activities were not affected.

In the present study, we examined the effect of the activation of the PKA system in vivo using a permeabilized-reactivated model of crayfish walking leg giant axon (Hirokawa and Yorifuji, 1986; Forman et al., 1987). It provides a good model system because it lacks neurofilament protein (Burton and Hinkley, 1974; Hirokawa, 1986; Hirokawa and Yorifuji, 1986; Viancour et al., 1987). Neurofilament is a good substrate for PKA (Leterrier et al., 1981); its phosphorylation can induce a substantial change in the structure of the neuronal cytoskeleton, and the organelle transport was greatly perturbed (mostly stimulated both in number and velocity) as reported in Aplysia growth cones (Forscher et al., 1987) (R. Sato-Yoshitake, unpublished results). In the crayfish giant axon, however, no apparent change in the axonal cytoskeleton was observed even at an electron microscopic level. We can thus assess the effects of the activation of the PKA system on the axonal transport system more directly.

Quantitative video-enhanced light microscopic and electron microscopic observation of this model revealed that the activation of the PKA system selectively inhibited the anterograde vesicle transport, but the retrograde transport and the mitochondria transport were not affected. These effects by PKA activa- 
tion, coupled with the previous in vitro results (Sato-Yoshitake et al., 1992) and the corresponding biochemical results reported here, support the idea that decommission of kinesin at the axon terminal is performed by the release of kinesin through its phosphorylation.

\section{Materials and Methods}

Video-enhanced light microscopy of the isolated crayfish walking leg giant axon. As described in our previous paper (Hirokawa and Yorifuji, 1986), a giant motor axon dissected from crayfish (Procambarus clarkii) walking leg was mounted in a perfusion chamber filled with crayfish internal medium (CFIM; $280 \mathrm{mM} \mathrm{K}$-aspartate, $15 \mathrm{mM} \mathrm{NaCl}$, $15 \mathrm{~mm}$ HEPES, $5 \mathrm{~mm} \mathrm{MgCl}_{2}, 3 \mathrm{~mm}$ EGTA, $\mathrm{pH}$ 7.4) (Lieberman et al., 1981). It was observed with an Axiophot microscope (Zeiss, Oberkochen, Germany) equipped with a $63 \times$ NA 1.40 plan-apochromat objective (Zeiss), an NA 1.4 condenser (Zeiss), and the G. W. Ellis fiberoptic light scrambler (Technical Vidco, Woods Holc, MA). The DIC image was projected to a video camera, Hamamatsu C-2400-07, by $6 \times$ relay lenses (Zeiss). With an image processor Argus-10 (Hamamatsu Photonics, Hamamatsu, Japan), the image of moving organelles was contrast enhanced by differentially subtracting rolling-averaged video frames. For quantification of the motility of the organelles, the objective focal plane was adjusted to the center of the axon, a line perpendicular to the long axis of the axon was drawn on the video monitor, and the number of moving organelles crossing the line was counted for $10 \mathrm{sec}$ (vesicles) or $5 \mathrm{~min}$ (mitochondria).

Application of the pharmacological probes to the permeabilized-re activated model and the quantification of its effect by video-enhanced light microscopy. Before the application of the pharmacological probes; the isolated axon was observed as described above, and the number of moving organelles was counted. Only the axons morc than $20 \mu \mathrm{m}$ in diameter and with more than 100 anterogradely moving vesicles were used for the following assays. In some preparations, the organelles in axons showed less smooth movement than other preparations. Such axons were discarded, because this type of movement was often observed in injured axons.

Membrane-permeable probes such as dbcAMP, KT5720, okadaic acid, and calyculin A were directly perfused into the chamber, and the number of moving organelles was counted.

For the application of the membrane-nonpermeable probes such as the catalytic subunit of PKA and ATP $\gamma \mathrm{S}$, the permeabilized-reactivated model was used. The axolemma was permeabilized by $30 \mathrm{~min}$ incubation in CFIM containing $0.015 \%$ saponin and $2 \mathrm{mM}$ ATP. When necessary, an ATP regeneration system ( $3 \mathrm{~mm}$ creatine phosphate, $1 \mathrm{unit} / \mathrm{ml}$ creatine phosphokinase) was added to this medium to maintain an optimum concentration of ATP. Then the permeabilizing medium containing PKA or other probes was perfused into the chamber, and the number of moving organelles was counted as above.

Quantification of the effect of PKA application by electron microscopy. The isolated giant axon was permeabilized and reactivated as described. After 30 min permeabilization, the catalytic subunits of PKA and ATP $\gamma \mathrm{S}$ were added at final concentrations of $0.05 \mathrm{mg} / \mathrm{ml}$ and 0.02 $\mathrm{mM}$, respectively. The sample was incubated for $35 \mathrm{~min}$ at $25^{\circ} \mathrm{C}$, and the midpoint of the axon was ligated with surgical thread. The axon was incubated for $5 \mathrm{~min}$ and fixed with $4 \%$ paraformaldehyde and $2 \%$ glutaraldehyde, followed by a conventional electron microscopic method. Thin cross sections were cut from the portion $20-60 \mu \mathrm{m}$ proximal or distal to the region damaged by the ligation. Electron micrographs of 200 axons from nine independent preparations for cach condition were taken. Two different authors independently examined the resulting 1200 micrographs in a blinded fashion. Each author scored the degree of accumulation of vesicles and mitochondria according to a predetermined scale $(0=$ no accumulation, $1=$ slight accumulation, $2=$ significant accumulation, $3=$ most accumulated), and the scores by the two authors were summed for each micrograph. Thus, the degree of organelle accumulation was semi-quantitatively scored by this "accumulation score," and histograms were plotted to illustrate changes in the degree of accumulation by shifts in their peaks. For the statistical evaluation of the shifts, the accumulated $\chi^{2}$ test was performed.

Detection of phosphorylated proteins in the axoplasm of the crayfish giant axon. Nerve fibers isolated from crayfish walking legs were squeezed with a handmade small roller onto a parafilm-coated petri dish. About 2-3 $\mu \mathrm{l}$ of axoplasm was obtained from one nerve fiber, which was diluted to $30 \mu \mathrm{l}$ with CFIM and centrifuged at $2000 \times \mathrm{g}$ for 10 min to remove contaminating axolemma debris. This axoplasm contained many microtubules and membranous organelles such as mitochondria and small and large vesicles. ATP (final $2 \mathrm{mM}$ ) and $\gamma^{-{ }^{32} \mathrm{P}-\mathrm{ATP}}$ (final $0.3 \mathrm{mCi} / \mathrm{ml}$ ) were added and incubated for $45 \mathrm{~min}$ in the presence or absence of the catalytic subunit of PKA (final $0.05 \mathrm{mg} / \mathrm{ml}$ ). The samples were analyzed on a $7.5 \%$ SDS-polyacrylamide gel (SDSPAGE) by Coomassie stain and autoradiography. The autoradiogram was quantitatively analyzed with an image analyzer BAS2000 (Fuji, Tokyo, Japan).

Purification of CF-kinesin from crayfish nervous tissue. CF-kinesin was purified from crayfish nerves and ganglia by scaling down the method already reported (Vale et al., 1985a,b; Wagner et al., 1989; SatoYoshitake et al., 1992). Briefly, walking leg nerves and abdominal ganglia were isolated from 300 crayfishes and homogenized in $10 \mathrm{ml}$ PEM (PIPES $100 \mathrm{~mm}, \mathrm{pH} 6.9$, EGTA $1 \mathrm{~mm}, \mathrm{MgCl}_{2} 1 \mathrm{~mm}$, dithiothreitol 1 $\mathrm{mm}$, phenylmethylsulfonylfluoride $10 \mu \mathrm{M}$, and leupeptin $10 \mu \mathrm{g} / \mathrm{ml}$ ). Microtubules, reconstituted from the clarified homogenate in the presence of $10 \mu \mathrm{M}$ taxol and $1 \mathrm{mM}$ ATP, were removed by centrifugation, and the supernatant was incubated with $10 \mathrm{U} / \mathrm{ml}$ hexokinase and $50 \mathrm{~mm}$ glucose for $5 \mathrm{~min}$ at $25^{\circ} \mathrm{C}$. The sample was further incubated with 2 $\mathrm{mM}$ adenylyl-5'-imidodiphosphate, $10 \mu \mathrm{M}$ taxol, and $0.3 \mathrm{mg} / \mathrm{ml}$ tubulin, and reconstituted microtubules were pelleted by centrifugation through a 50\% sucrose cushion. This pellet was extracted with $1 \mathrm{ml}$ PEM containing $10 \mathrm{~mm} \mathrm{MgATP}$ on ice, and microtubules were removed by centrifugation with $20 \mu \mathrm{M}$ taxol. The supernatant was dialyzed against TM (50 $\mathrm{mm}$ Tris, $\mathrm{pH} \mathrm{7.2,} \mathrm{and} 1 \mathrm{~mm} \mathrm{MgCl}_{2}$ ), and this fraction was used in the following assays.

Metabolic labeling of $C F$-kinesin and peptide mapping. Carefully dissected ventral nerve cord was incubated in CFIM supplemented with 1 $\mathrm{mCi} / \mathrm{ml}{ }^{32} \mathrm{P}$-orthophosphate for $30 \mathrm{~min}$. CF-kinesin was partially purified as described above, and the $120 \mathrm{kDa}$ band was excised from SDSPAGE gel. At the same time, purified CF-kinesin was phosphorylated in vitro by $60 \mathrm{~min}$ incubation with $0.1 \mathrm{mM}$ ATP containing $0.3 \mathrm{mCi} / \mathrm{ml}$ $\gamma{ }^{32} \mathrm{P}$-ATP and $0.05 \mathrm{mg} / \mathrm{ml}$ catalytic subunit of PKA, and excised from SDS-PAGE gel. These in vivo and in vitro phosphorylated CF-kinesin were partially digested by V8 protease and analyzed by a second $15 \%$ SDS-PAGE gel as described by Cleveland et al. (1977).

Assay of phosphatase activity in axoplasm. Purified CF-kinesin $(0.1$ $\mathrm{mg} / \mathrm{ml}$ ) was phosphorylated by $60 \mathrm{~min}$ incubation with $0.1 \mathrm{mM}$ ATP containing $0.3 \mathrm{mCi} / \mathrm{ml} \gamma_{-}{ }^{32} \mathrm{P}-\mathrm{ATP}$ and $0.05 \mathrm{mg} / \mathrm{ml}$ catalytic subunit of PKA. CF-kinesin and two contamination bands (70 kDa protein and tubulin) were strongly phosphorylated. Excess cold ATP (10 mM), phosphatase inhibitors (okadaic acid and calyculin A), and protease inhibitors were added to this sample, and it was incubated in the presence or absence of the extruded axoplasm (1:4 v:v), and analyzed on a $7.5 \%$ SDS-PAGE by Coomassie stain and autoradiography. The autoradiogram was quantitatively analyzed with a BAS2000 image analyzer (Fuji, Tokyo, Japan).

Association of axonal organelles and kinesin. Extruded axoplasm from six nerve fibers was diluted 1:1000 with ice-cold CFIM and centrifuged at $16,000 \mathrm{rpm}$ for $30 \mathrm{~min}$ by desk-top centrifuge after $30 \mathrm{~min}$ incubation at $-1{ }^{\circ} \mathrm{C}$. The supernatant was observed by video-enhanced light microscopy and negative-staining electron microscopy, and it was confirmed that its major component was membranous organelles other than mitochondria or the debris of axolemma, and that it contained little cytoskeletal filamentous structure. Otherwise this centrifugation step was repeated. The organelles were collected by centrifugation (Beckman TL100.2 rotor, 75,000 rpm, $30 \mathrm{~min}$ ) and the pellet was resuspended with $100 \mu \mathrm{l} \mathrm{TM}$ containing $500 \mathrm{mM} \mathrm{KCl}$, as previously described (SatoYoshitake et al., 1992), to remove kinesin already bound to the organelles. The sample was again centrifuged and the pellet was resuspended with $50 \mu \mathrm{l}$ TM. This sample was checked by video-enhanced light microscopy and negative-staining electron microscopy, and many small and large vesicular organelles and occasionally mitochondria were observed, but microtubules or the debris of axolemma were rarely found.

Phosphorylated $\mathrm{CF}$-kinesin was obtained by $60 \mathrm{~min}$ incubation of the purified CF-kinesin $(0.1 \mathrm{mg} / \mathrm{ml})$ with the catalytic subunit of PKA $(0.05$ $\mathrm{mg} / \mathrm{ml}$ ) and $2 \mathrm{mM}$ ATP at $25^{\circ} \mathrm{C}$. For unphosphorylated CF-kinesin, the purified CF-kinesin was incubated under the same conditions but without PKA. The CF-kinesin aliquots were centrifuged (Beckman TL100.2 rotor, $100,000 \mathrm{rpm}, 30 \mathrm{~min}$ ) to remove the aggregates.

The organelles were added to the kinesin aliquots and incubated for $30 \mathrm{~min}$ at $25^{\circ} \mathrm{C}$. For the control, samples without organelles or kinesin were incubated under the same conditions. The samples were then centrifuged (Beckman TL100.2 rotor, 100,000 rpm, $30 \mathrm{~min}$ ) to separate the 
bound/free kinesin. In this step, cosedimentation of free kinesin with contaminating microtubules and its nonspecific binding to the tube surface can obscure the result. Therefore, we performed the centrifugation in the presence of ATP to reduce the binding of kinesin to microtubules, and siliconized tubes were used to prevent nonspecific binding. The pellets and the supernatants were analyzed by immunoblotting using monoclonal anti-kinesin antibody $\mathrm{H} 2$ and ${ }^{125}$ I-labeled protein $\mathrm{A}$ (ICN, Tokyo, Japan). The results were quantified by analyzing the autoradiograms of the blot with BAS2000 (Fuji, Tokyo, Japan).

Other materials and reagents. The catalytic subunit of PKA, monoclonal anti-kinesin heavy chain antibody $\mathrm{H} 2$, and taxol were generous gifts from Dr. M. Inagaki (Tokyo Metropolitan Institute of Gerontology), Dr. G. S. Bloom (University of Texas), and Dr. N. Lomax (National Cancer Institute), respectively. Crayfish was purchased from a local distributor. Nucleotides, nucleotide analogs, and detergent were purchased from Sigma (St. Louis, MO). Buffers were purchased from Dojindo Laboratories (Kumamoto, Japan). Other reagents were purchased from Wako Chemical (Tokyo, Japan) unless otherwise stated.

\section{Results}

Observation of isolated giant axon by video-enhanced light microscopy

$\Lambda s$ in our previous study (Hirokawa and Yorifuji, 1986), two distinct types of organelles were observed to move both anterogradely and retrogradely in the isolated crayfish walking leg giant axon observed by video-enhanced light microscopy. One is a long $(\sim 1 \mu \mathrm{m})$ large organelle moving slowly $(\sim 0.2 \mu \mathrm{m} / \mathrm{sec})$ in a saccadic manner. The other is a small (smaller than the diffraction limit), spherical organelle moving rapidly (1-2 $\mu \mathrm{m} / \mathrm{sec}$ ) in a smooth manner. From their shapc and sizc, the former is considered to be a mitochondrion, and the latter is assumed to be a vesicle. This interpretation was confirmed by the results from the electron microscopic observations described in the following section. Hence, to avoid unnecessary complexity, we refer to the smaller, spherical organelles as vesicles, and the larger, longer organelles as mitochondria in the following sections describing the results of light microscopic observations.

A quantitative measure is necessary for the evaluation of the drug effect. A naive one is the number of moving organelles. Indeed, it was maintained around the same level $(\mathrm{SD} \sim 5 \%$ of the mean) for more than $2 \mathrm{hr}$ in the control conditions, but it varied very much (SD $\sim 50-100 \%$ of the mean) from preparation to preparation (Fig. 1a,c; see Fig. $4 a-d$, CTRL traces), which made it difficult to compare different preparations. Preliminary studies, however, suggested the response to the drug was proportional to the initial level. Therefore we used the relative changes in the number of moving organelles as the measure for the drug effect. For this purpose, "relative motility index" was calculated by dividing the number of moving organelles by the average before drug application. In any conditions in this study, the SD of this index was within $10 \%$ of the mean (mostly 3-5\%), much smaller than that of raw number (50-100\%) and small enough to detect the drug effects in this study.

\section{Application of dbcAMP selectively inhibits anterograde small vesicle transport}

For the activation of the PKA system, we first applied dbcAMP to the nonpermeabilized isolated giant axon. DbcAMP is a membrane-permeable analog of cAMP, and activates PKA. After the addition of dbcAMP, the number of anterogradely transported vesicles decreased (Fig. $1 b, e$ ), while the number of retrogradely transported vesicles and anterogradely or retrogradely transported mitochondria showed no decrease (Fig. $1 d, f-h$ ). The velocity of transported organelles did not change. The inhibition of the anterograde vesicle transport was transient, reaching its peak at around $30 \mathrm{~min}$ atter dbcAMP application, and then recovering to the initial level at 40-60 min after dbcAMP application, even when new medium containing the same concentration of dbcAMP was perfused into the chamber. This indicated that the recovery was not due to the consumption of dbcAMP.

The inhibition by dbcAMP was concentration dependent (Fig. $2 a$ ). At $1 \mathrm{~mm}$, the anterograde vesicle transport was most inhibited. The higher the concentration, the more rapidly the inhibition reached its peak and recovered (Fig. 1e). It appears that increasing the concentration of dbcAMP stimulated both the inhibition and its recovery.

To demonstrate that PKA is responsible for this inhibition by dbcAMP, we used a specific PKA inhibitor:KT5720. When KT5720 was applied to the medium, the inhibition of the anterograde vesicle transport by $1 \mathrm{mM}$ dbcAMP was reversed in a dose-dependent manner, while KT5720 alone showed no effects on organelle transport (Fig. le). $\mathrm{IC}_{50}$ for this inhibition was $\sim 50$ nM. It corresponds well with the $\mathrm{IC}_{50}$ for the inhibition of PKA, and was much lower than the $\mathrm{IC}_{50}$ s for the inhibition of other kinases such as protein kinase $\mathrm{C}, \mathrm{Ca}^{2+}$-calmodulin kinase, and myosin light chain kinase (Kase et al., 1987). This demonstrates that PKA plays a key role in the effect of dbcAMP on organelle transport.

The selective inhibition by $d b c A M P$ is caused by the activation of $P K A$

To study further the mechanism of the inhibition by dbcAMP, we used a permeabilized-reactivated model. In this model, most cytosolic soluble proteins are washed out, and only cytoskeletal and membrane-associated components remain.

Using this model, we first evaluated what effect the decrease or increase of ATP concentration might have with the organelle motility, because organelle motility depends on ATP and its depletion ceases the motility. In fact, in preliminary experiments without $\Lambda$ TP regeneration system, activation of PKA pathway depleted ATP and organelle movement stopped. This is why we included ATP regeneration system in the reactivation medium, but still there remains a possibility that a slight decrease of ATP concentration by pharmacological perturbation can influence organelle movement.

To rule out this possibility, the ATP concentration in the reactivation medium was altered, and organelle motility was observed. As shown in Figure 3, both anterograde and retrograde vesicle transports decreased concentration dependently when the ATP concentration was lower than $1 \mathrm{mM}$. The retrograde transport was more sensitive to the decrease of ATP concentration than the anterograde one. Therefore, anterograde-specific inhibition cannot be reproduced by decreasing ATP concentration; that is, the observed specific inhibition of anterograde vesicle transport is not caused by the possible decrease of ATP concentration by pharmacological perturbation, but rather by its pharmacological actions.

Second, we applied the catalytic subunit of PKA to the permeabilized-reactivated model. To maintain the concentration of ATP, an ATP regeneration system was supplementarily added to the reactivation medium, and the concentration of ATP was kept at 1-2 $\mathrm{mM}$, the optimum concentration for organelle transport (Fig. 3). The effect of the application of PKA was similar to that by dbcAMP. The anterograde vesicle transport showed a selective and transient decrease in number (Fig. $4 a, e$ ) but not in velocity, while no significant changes were observed in the ret- 

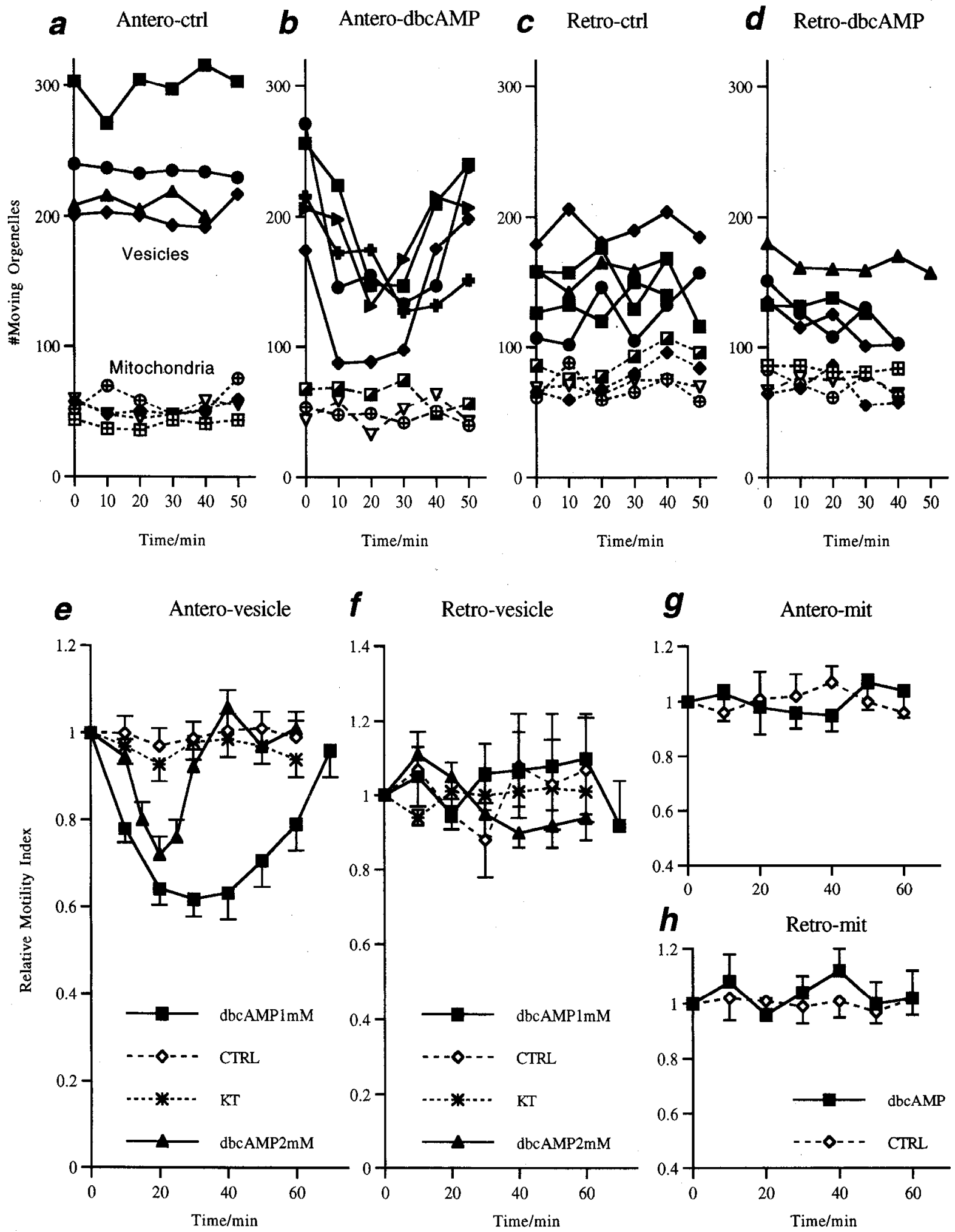

Figure 1. Effects of dbcAMP on organelle transport in the crayfish giant axon. The numbers of anterogradely $(a, b)$ and retrogradely $(c, d)$ moving organelles were counted in the absence $(a, c)$ and presence $(b, d)$ of dbcAMP $(1 \mathrm{~mm})$. Some typical raw data are plotted against time after the drug application in $a-d$. Open symbols with dotted lines indicate the number of moving mitochondria, and closed symbols with solid lines show that of small vesicles. These raw data were transformed into "relative motility indices" by dividing the average values before the drug application, and the mean and SEM of eight independent preparations are plotted in $e-h(e$, anterograde small vesicles; $f$, retrograde small vesicles; $g$, anterograde mitochondria; $h$, retrograde mitochondria). The values in the absence of dbcAMP $(\diamond)$ and in the presence of 1 mM dbcAMP ( $)$ are indicated in $e-h$, and those in the presence of $2 \mathrm{mM} \mathrm{dbcAMP}(\mathbf{\Lambda})$ and in the presence of $1 \mathrm{mM}$ dbcAMP and $2 \mu \mathrm{M}$ KT5720 (*) are also shown in $e$ and $f$. The application of dbcAMP caused a selective and transient reduction in the number of anterogradely transported small vesicles, and this reduction was reversed by the application of the specific PKA inhibitor KT5720. 
a

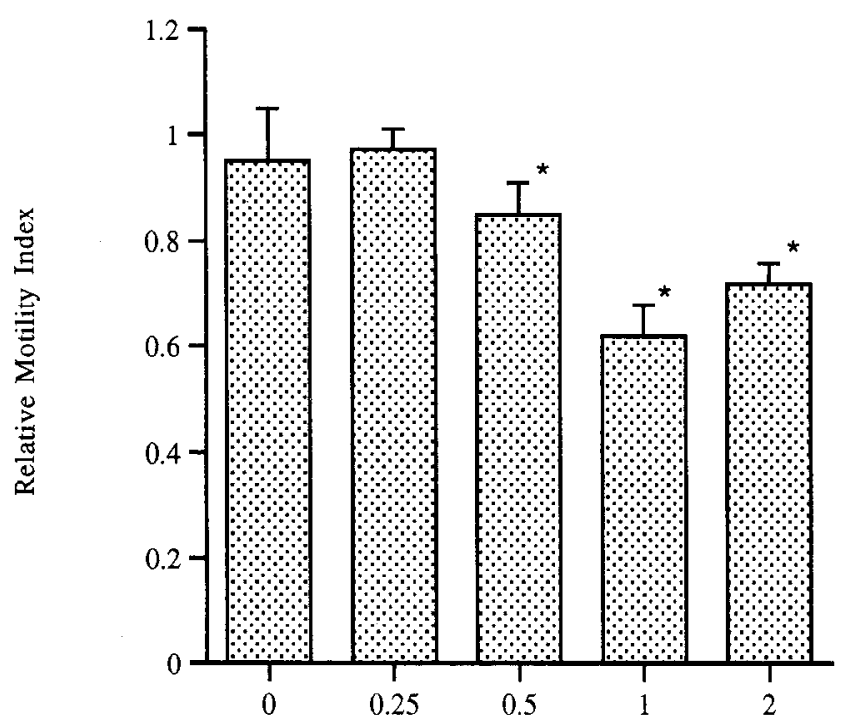

$\mathrm{dbcAMP} / \mathrm{mM}$
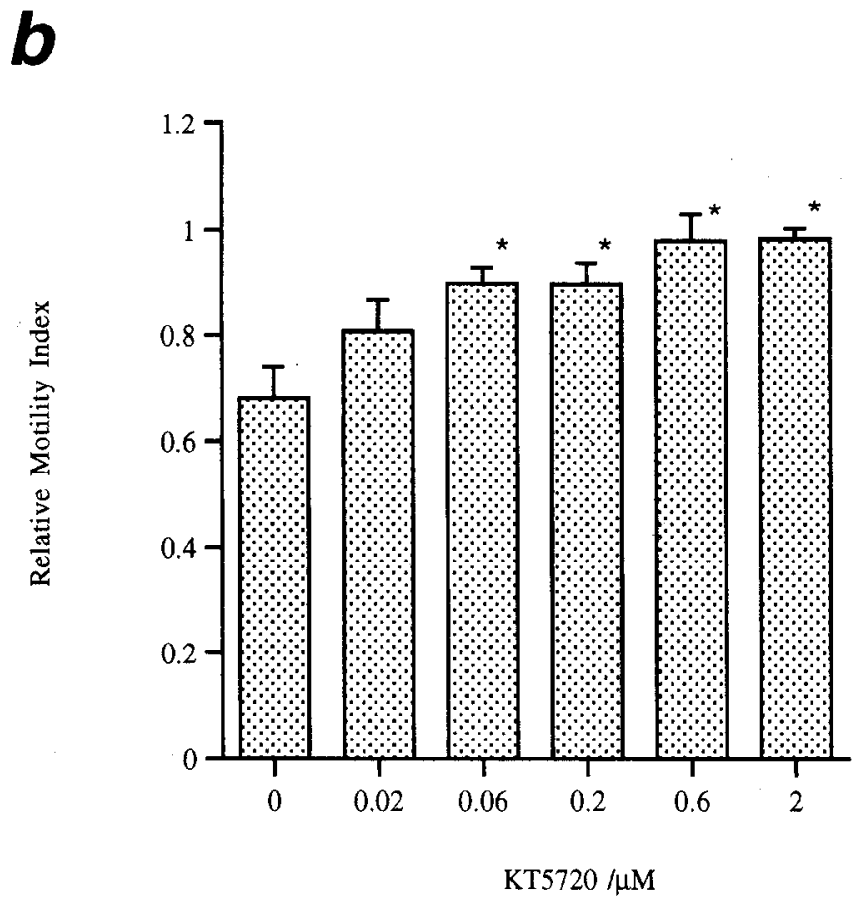

Figure 2. Dose dependence of the reduction of the anterograde vesicle transport by dbcAMP $(a)$ and its reversal by KT5720 $(b)$. Mean and SEM of relative motility indices at 25 min after the drug application from five independent experiments are shown. Asterisks indicate that the reduction or its reversal was significant at $P<0.05$ by Student's $t$ test.

rograde vesicle transport or mitochondria transport (Fig. $4 b, f$ ). These results support the interpretation that the anterograde-specific inhibition by dbcAMP is due to the activation of PKA, and that the inhibition is not due to the change of ATP concentration in the axoplasm.

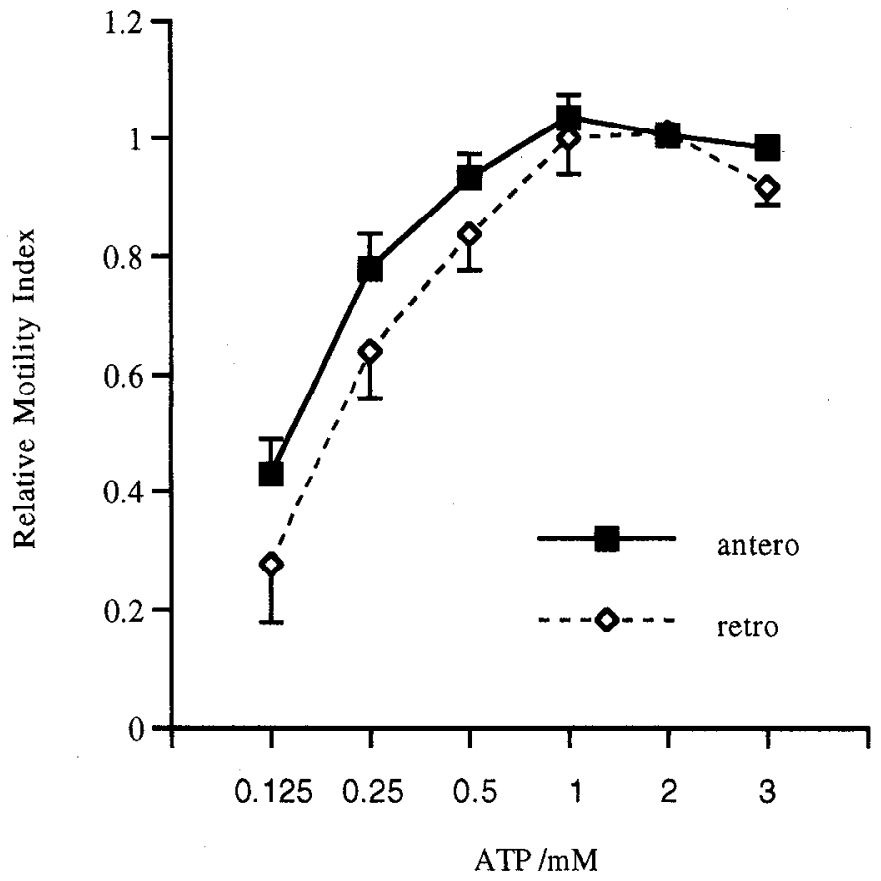

Figure 3. The effects of the alteration of ATP concentration on the anterograde $(\square)$ and retrograde $(\diamond)$ vesicle transport. Mean and SEM from three independent experiments are shown. Reduction of ATP concentration has an inhibitory effect on both the anterograde and retrograde transport, but the latter was more sensitive. This difference in sensitivity was significant at $P<0.01$ by the paired $t$ test. Note that at any concentration of ATP, the relative motility index for the anterograde transport was equal to or greater than that for the retrograde one.

\section{ATP $\gamma S$ augments the inhibition by PKA}

The application of dbcAMP or PKA showed a biphasic effect on vesicle transport: inhibition and its recovery. One possible explanation for the recovery is that protein phosphatase dephosphorylates a protein or proteins once phosphorylated by PKA. To inspect this possibility, okadaic acid and calyculin A, broad range phosphatase inhibitors, were used. Neither of them showed a significant effect; these drugs did not interfere with the inhibition by PKA.

However, there still remains room for a possible role of protein phosphatase, for only protein phosphatases $1,2 \mathrm{~A}$, and $2 \mathrm{~B}$ are inhibited by these drugs. Then, to inhibit all the phosphatases, the ATP analog ATP $\gamma$ S was applied. It can be the substrate for the kinase and the target protein is thiophosphorylated, but the thiophosphate moiety is hardly hydrolyzed by protein phosphatase. Because Shimizu et al. (1991) have reported that ATP $\gamma \mathrm{S}$ inhibits vesicle transport and motor proteins such as kinesin at higher concentrations, we carefully determined the concentration of ATP $\gamma S$ to avoid its inhibitory effects on the transport. No significant effect by ATP $\gamma S$ was observable up to 200 $\mu \mathrm{M}$ even after $120 \mathrm{~min}$ incubation in the medium (Fig. 4, CTRL traces).

In the presence of $100 \mu \mathrm{M}$ ATP $\gamma \mathrm{S}$, however, the effect of the catalytic subunit of PKA was augmented. The anterograde vesicle transport was suppressed in number (Fig. $4 a, e$ ) but not in velocity, and the retrograde vesicle transport (Fig. $4 b, f$ ) and the mitochondria transport (Fig. $4 c, d, g, h$ ) were not inhibited. The decrease in the number of anterogradely transported organelles was greater than in the absence of ATP $\gamma \mathrm{S}$, and the recovery was 

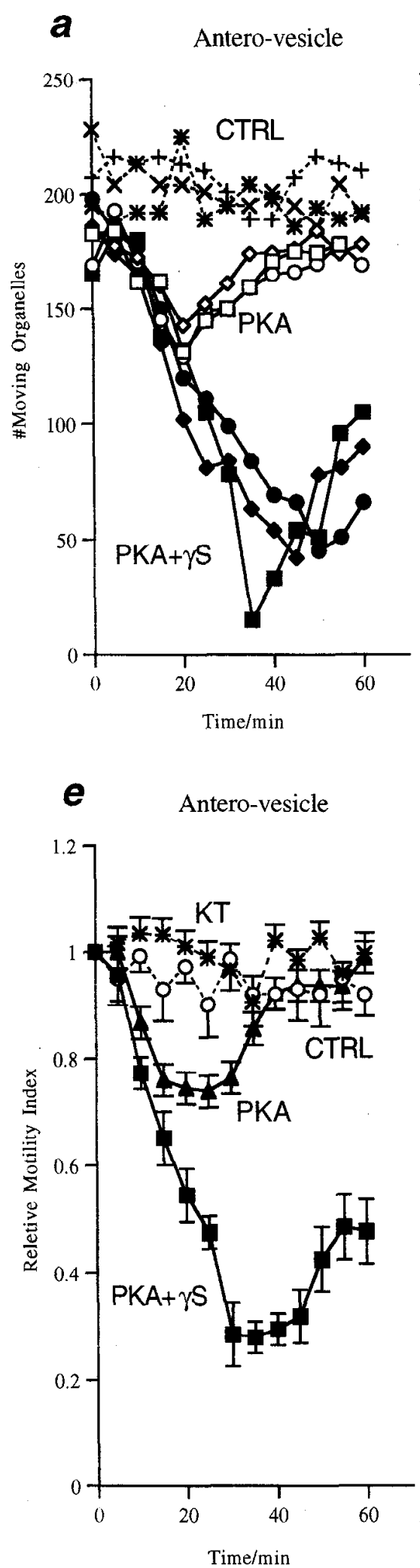

$\boldsymbol{b}$

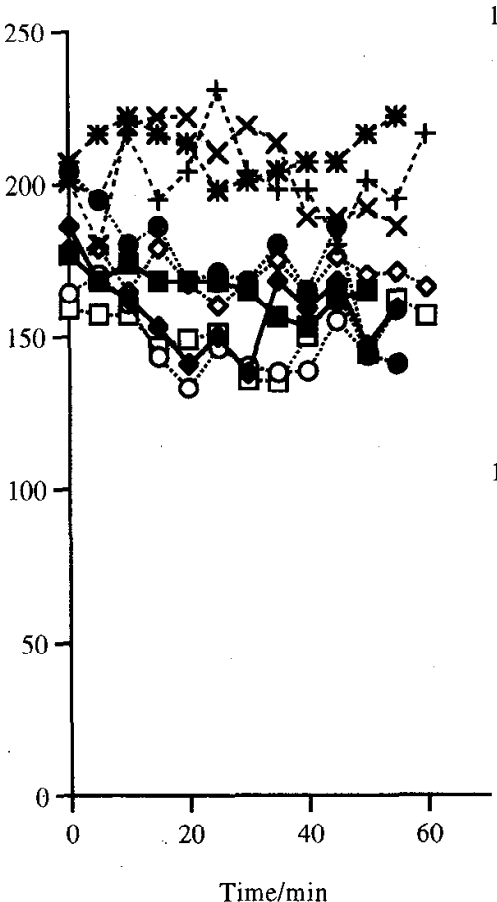

Time/min

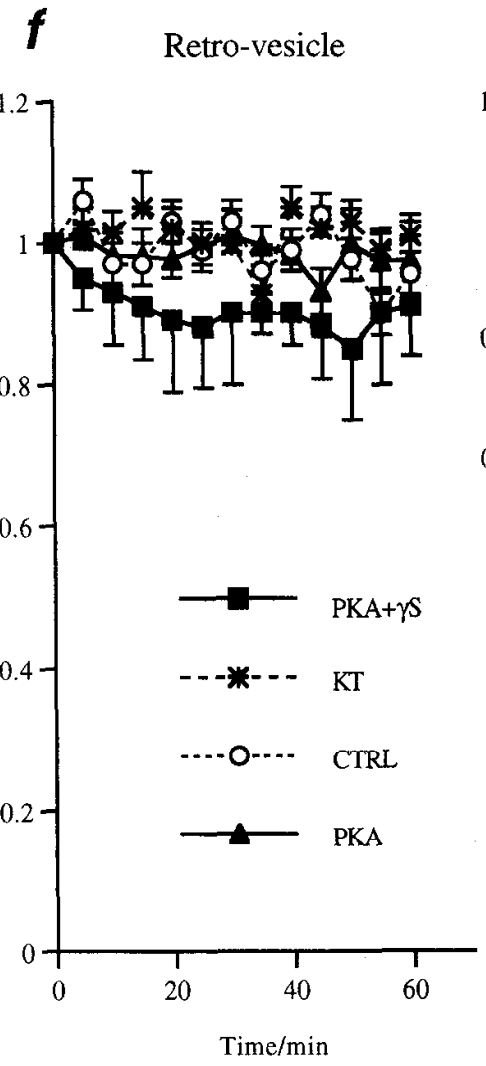

C Amtero-mit
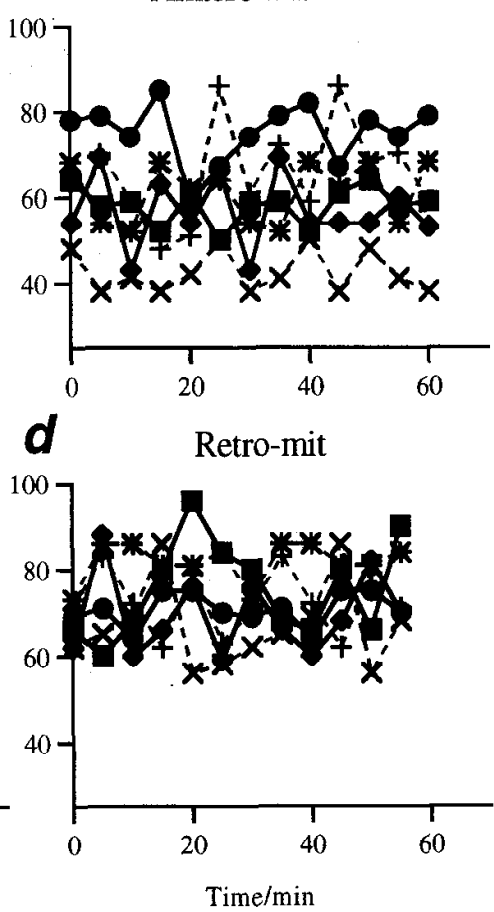

$9 \quad$ Antero-mit
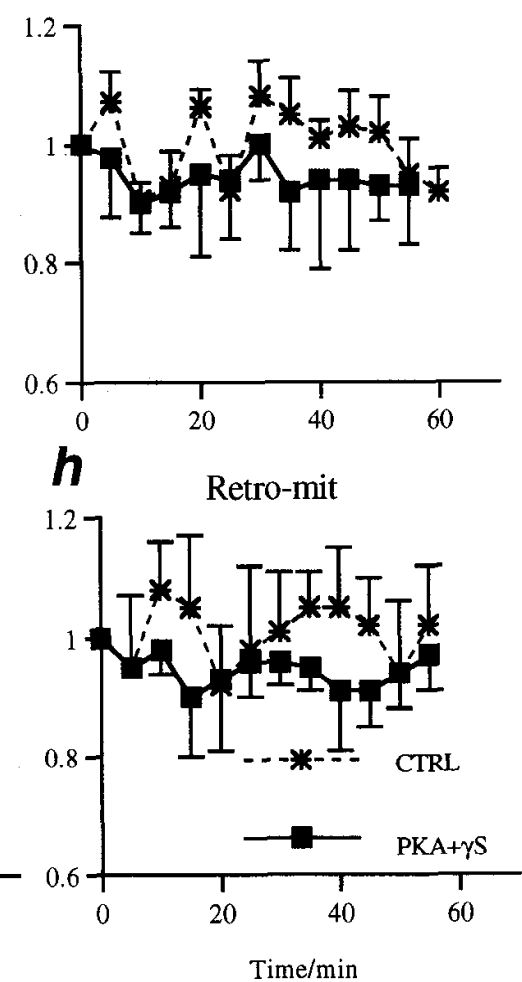

Figure 4. The effects of the application of the exogenous PKA catalytic subunit to the permeabilized-reactivated model of crayfish giant axon. $a-d$ show typical raw data, and the mean and SEM of relative motility indices from eight independent preparations are plotted against time after PKA application $(e-h) . C T R L, P K A, P K A+\gamma S$, and $K T$ indicate the conditions: $C T R L=\mathrm{ATP} \gamma \mathrm{S}$ only; $P K A=\mathrm{PKA}$ only; $P K A+\gamma S=\mathrm{PKA}$ and $\mathrm{ATP} \gamma \mathrm{S}$; and $K T=\mathrm{PKA}, \mathrm{ATP} \gamma \mathrm{S}$, and $\mathrm{K} 15720$. The application of the exogenous PKA catalytic subunit transiently and selectively inhibited the anterograde vesicle transport ( $P<0.01$ by $t$ test at $15-30$ min after PKA application). This effect was augmented by the addition of ATP $\gamma$, and its recovery was inhibited $(P<0.01$ at $30-60 \mathrm{~min})$. 
inhibited in the presence of ATP $\gamma \mathrm{S}$ (Fig. 4a,e). This suggests the involvement of phosphatase activity in the recovery phase.

\section{EM observation confirmed that PKA-sensitive organelles are small vesicles, but not mitochondria}

Contrast-enhanced light microscopy revealed that the anterograde organelle transport can be divided into two pharmacologically different systems: one is sensitive to PKA activation and the other is insensitive or less sensitive. The limited resolution of light microscopy enabled us to discriminate only two morphologically different populations. One consisted of small, spherical, smoothly moving organelles whose movement was specifically inhibited by the activation of the PKA system, and the other was made up of larger, longer, saltatory moving organelles that were not inhibited by PKA.

For observation at a higher resolution, electron microscopy was used. Ligation of the axon is an effective method for discriminating the anterogradely or retrogradely transported organelles from nontransported ones (Smith, 1980; Hirokawa et al., $1990,1991)$. The anterogradely transported organelles accumulate at the proximal portion to the ligated site, while the retrograde ones accumulate at the distal portion. Nontransported organelles do not accumulate.

The permeabilized-reactivated giant axon was ligated after 35 min incubation in the presence of ATP $\gamma \mathrm{S}$ and the catalytic subunit of PKA, and was fixed $5 \mathrm{~min}$ after its ligation. This preparation allowed us to ubserve only the organelles noving during the period 35-40 min after PKA application, when the inhibition of the transport reached its peak (Fig. 4a,e). Typical electron micrographs are demonstrated in Figure 5. First of all, unlike mammalian axons, no apparent changes of the cytoskeletal organization were observed with these treatments (Fig. $5 a-f$ ). Under control conditions (Fig. $5 a, d$ ), many vesicles and mitochondria accumulated at both proximal and distal regions. Some accumulated vesicles fused to form large membranous cisternae. The addition of PKA caused a decrease in the accumulation of vesicular and membranous cisternae at the proximal region (Fig. $5 b$ ), while the accumulation of mitochondria showed no apparent change, and no distinct change was observed at the distal region (Fig. 5e).

The effects of the application of the pharmacological probes on the accumulation of organelles at both the proximal and distal regions were semi-quantitatively analyzed. As shown in Figure $5 g-j$, the degree of accumulation was scored and the histogram was calculated. No significant difference was detected in the distal portion (Fig. $5 h, j$ ), as expected from the results by light microscope. At the proximal region, the accumulation of vesicles and membranous cisternae was significantly inhibited, and this inhibition was reversed by the application of the PKA inhibitor KT5720 (Fig. $5 g$ ), while the accumulation of mitochondria was not affected (Fig. 5i).

Thus, the observation at the electron microscopic level confirmed the results obtained by light microscopy that the activation of the PKA system selectively inhibited the anterograde transport of vesicles.

\section{Kinesin is a possible target of PKA}

The activation of PKA system in crayfish giant axon specifically inhibits the anterograde vesicle transport. Then what is the target of PKA? To answer this question, we searched a protein band(s) that was specifically phosphorylated by the activation of PKA pathway. The phosphorylated proteins in the axoplasm were an- alyzed by SDS-PAGE and detected by autoradiography. At least 13 bands were identified on the autoradiogram (Fig. 6, lane 1'). Their radioactivities were significantly increased in the sample incubated in the presence of PKA (Гig. 6, lane $2^{\prime}$ ). The addition of KT5720 reversed this increase (Fig. 6, lane $3^{\prime}$ ). Thus these bands are gond candidates for the target of PKA.

Kinesin is reported to be a substrate of PKA in vitro (Murphy et al., 1989; Sato-Yoshitake et al., 1992), and several studies have revealed that kinesin is a phosphoprotein in vivo (Hollenbeck, 1993) and that its phosphorylation level is raised by PKA activation (Matthies et al., 1993). Furthermore, our previous paper (Sato-Yoshitake et al., 1992) suggested the possible role of kinesin phosphorylation in the regulation of the fast axonal transport. Thus kinesin is a good candidate for the substrate, and we performed the following experiments for its demonstration.

To clarify whether kinesin is really phosphorylated in crayfish giant axon, we purified crayfish kinesin (CF-kinesin) by nucleotide-dependent binding to and release from microtubules. The resulting fraction supported microtubule motility (data not shown). A $120 \mathrm{kDa}$ protcin was concentrated in this fraction (Fig. 6, lane 7), and this band was recognized by anti-kinesin heavy chain monoclonal antibody H2 (Pfister et al., 1989) (see Fig. 9). We therefore consider this $120 \mathrm{kDa}$ protein to be the kinesin heavy chain homolog, and we refer to it as CF-kinesin. This fraction was purer than the corresponding fraction from mammalian brain. Only tubulin and CF-kinesin were the major components, and other contaminating bands were very subtle (Fig. 6, lane 7). Thus we used this fraction in the following assays. We could not identify kinesin light chain (KLC) in this fraction, mainly due to the lack of anti-KLC antibody that crossreacts with crayfish.

This CF-kinesin fraction was added to the extruded axoplasm (Fig, 6, lanes 4-6). CF-kinesin was phosphorylated in vitro among axoplasmic proteins and the band around $116 \mathrm{kDa}$ was demonstrated to correspond to that of CF-kinesin (Fig. 6, lanes $\left.4^{\prime} 6^{\prime}\right)$.

\section{CF-kinesin is phosphorylated by PKA in vivo}

Thus CF-kinesin is a good candidate for the target of PKA in vivo, but is it really phosphorylated in nonperturbed crayfish neuron, and is it directly phosphorylated by PKA? To answer these questions, we metabolically labeled the ventral nerve ganglia with ${ }^{32} \mathrm{P}$-orthophosphate, purified $\mathrm{CF}$-kinesin from these ganglia, and performed phosphopeptide mapping to compare the in vivo phosphorylation site with the PKA phosphorylation site in vitro.

CF-kinesin was actually phosphorylated in vivo (Fig. 7a, lane $2^{\prime} ; b$, lanes 5-8), and the phosphopeptide mapping was identical with CF-kinesin phosphorylated in vitro by PKA (Fig. 7b). This demonstrates that CF-kinesin is a phosphoprotein in crayfish neuron, and that the $116 \mathrm{kDa}$ PKA substrate in the crayfish axoplasm is CF-kinesin.

\section{Crayfish axoplasm has a phosphatase activity specific to $C F$ - kinesin}

The results obtained with the contrast-enhanced light microscope suggested that the axoplasm has a phosphatase activity which dephosphorylates the target protein of PKA, and thus the inhibition of the anterograde vesicle transport was recovered.

This means that the target protein of PKA must be dephosphorylated by an axoplasmic factor. We therefore incubated phosphorylated CF-kinesin with extruded axoplasm. As shown 




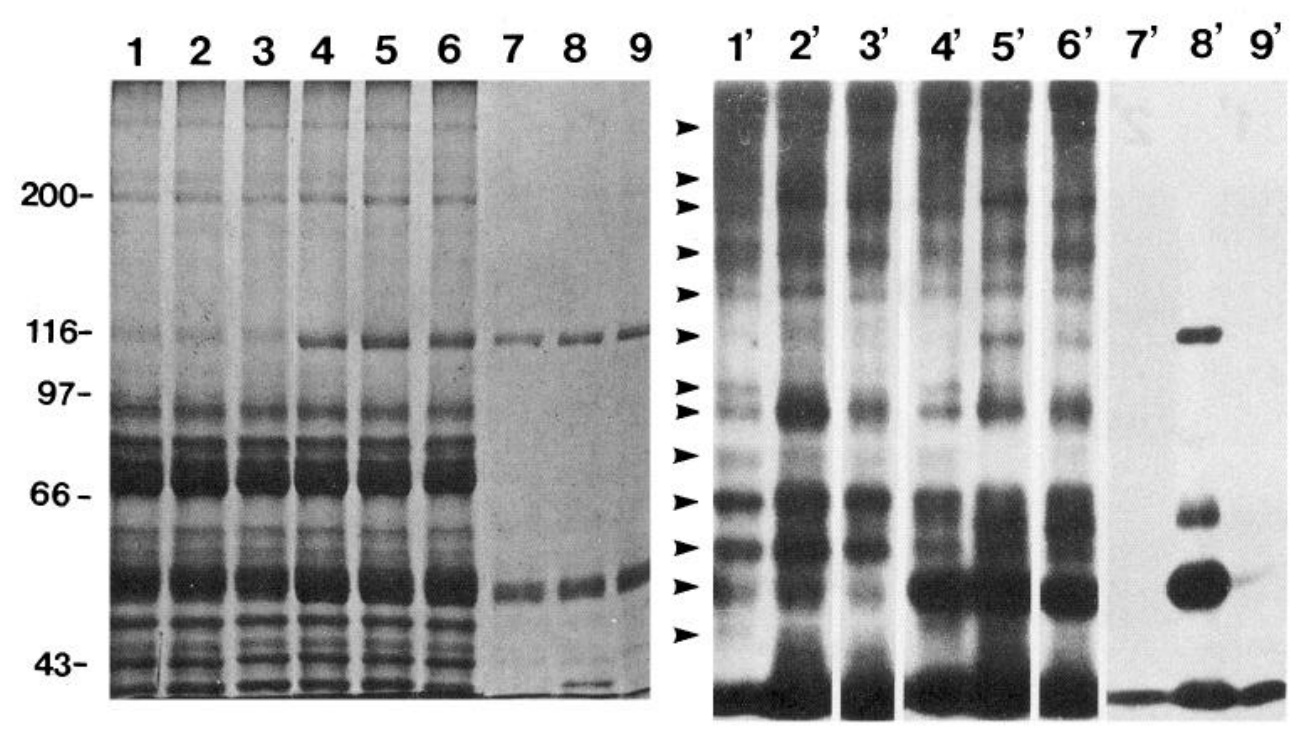

Figure 6. Phosphorylation of crayfish axoplasmic proteins by the application of PKA under the condition corresponding to that used for the microscopic observation. Extruded axoplasm (lanes $1-3$ ), extruded axoplasm supplemented with purified CF-kinesin (lanes 4-6), and purified CFkinesin (lanes 7-9) were incubated in the absence of PKA $(1,4,7)$, in the presence of PKA $(2,5,8)$, and in the presence of PKA and KT5720 $(3,6,9)$. The samples were analyzed on SDS-polyacrylamide gel by Coomassie stain $(I-9)$, and by autoradiography $\left(I^{\prime}-9^{\prime}\right)$. The positions of the molecular weight marker proteins are indicated on the left of lane 1 . At least 13 phosphoprotein bands were recognized in lanes $I^{\prime}$ and $2^{\prime}$, and their positions are indicated on the left of lane $1^{\prime}$. Application of PKA increased the level of phosphorylation of these bands (lane $\left.2^{\prime}\right)$, and this increase was reversed by KT5720 (lane $3^{\prime}$ ). Purified CF-kinesin was phosphorylated by PKA (lane $8^{\prime}$ ) even in the presence of other axoplasmic proteins (lane $5^{\prime}$ ). The position of the phosphorylated CF-kinesin band was around $116 \mathrm{kDa}$, corresponding to the phosphoprotein band around $116 \mathrm{kDa}$ in lane $2^{\prime}$.

in Figure 8, the amount of phosphate moiety incorporated into CF-kinesin decreased in a time-dependent manner even in the presence of okadaic acid or calyculin A, but no significant change was found in the two other contaminating phosphoproteins p70 and tubulin. Thus the axoplasm was demonstrated to have phosphatase activity which dephosphorylates phosphorylated kinesin, one of the possible targets of PKA. This phosphatase activity was not inhibited by okadaic acid or calyculin A, corresponding well with the observation by light microscopy.

\section{PKA phosphorylation of CF-kinesin reduces its affinity to axonal organelles}

The above results collectively show that the phosphorylation of CF-kinesin by PKA parallel the selective inhibition of anterograde axonal vesicle transport. Then can the phosphorylation of kinesin cause the inhibition? Several studies deny the possibility that phosphorylation of kinesin directly inhibits its motor activity (Murphy et al., 1989; Matthies et al., 1993). In fact, the microtubule motility by kinesin was not affected by its phosphorylation (data not shown).

The second possibility is that the phosphorylation uncouples kinesin from its cargo. Our previous in vitro study with rodent kinesin and organelle (Sato-Yoshitake et al., 1992) supports this possibility. To confirm that this is also the case with CF-kinesin, we performed a binding experiment with crayfish axoplasmic organelles and CF-kinesin.

Crayfish axoplasmic organelles were collected from the extruded axoplasm to avoid the contamination of axolemma. Membrane organelles were collected by sequential centrifugation. Mitochondria were mostly removed by the first centrifugation; most microtubules, the only major filamentous cytoskeleton, were depolymerized by the dilution and chilling just above freezing point; and most nondepolymerized microtubules were also removed by the first centrifugation. Thus the pellet of the second centrifugation contained little cytoskeletal filamentous structure.

After washing with salt to remove already binding kinesin, this organelle fraction was mixed with purified CF-kinesin, and the amount of free/bound kinesin was measured by quantitative immunoblotting with anti-kinesin heavy chain $\mathrm{H} 2$ antibody (Pfister et al., 1989). As expected, the binding of CF-kinesin to the axoplasmic organelles decreased to $75 \pm 5 \%$ (mean \pm SEM, $N=5$ ) after the phosphorylation of CF-kinesin by PKA (Fig. 9). This supports the idea suggested in our previous in vitro study that PKA phosphorylation of kinesin inhibits organelle motility by releasing the motor from its cargo, though the ob-

$\leftarrow$

Figure 5. Accumulation of transported organelles proximal $(a-c)$ and distal $(d-f)$ to the ligation. In the control group (ATP $\gamma \mathrm{S}$ only), at both proximal $(a)$ and distal $(d)$ portions, many mitochondria (arrow) and vesicles (small arrowhead) accumulated, and accumulated vesicles fused to form large membranous cisternae (large arrowhead). After application of PKA and ATP $\gamma \mathrm{S}$, the accumulation of vesicles decreased at the proximal portion $(b)$, while that at the distal portion $(e)$ and the accumulation of mitochondria had no apparent changes. The decrease of vesicle accumulation at the proximal portion was inhibited by the addition of KT5720 ( , proximal portion; $f$, distal portion). $g-j$ show the histograms of the degree of accumulation scored as described in Materials and Methods ( $g$, proximal-vesicle; $h$, distal-vesicle; $i$, proximal-mitochondria; $j$, distal-mitochondria). The application of PKA $(\times)$ selectively reduced the accumulation of vesicles at the proximal portion, which caused a significant shift of the peak in $g\left(P<0.01\right.$ by accumulated $\chi^{2}$ test). This reduction was reversed to the control level ( ) by the addition of KT5720 ( $\left.\square\right)$. No significant change in the profile of these histograms was detected in distal portions $(h, j)$ or in the accumulation of mitochondria $(i, j)$. 


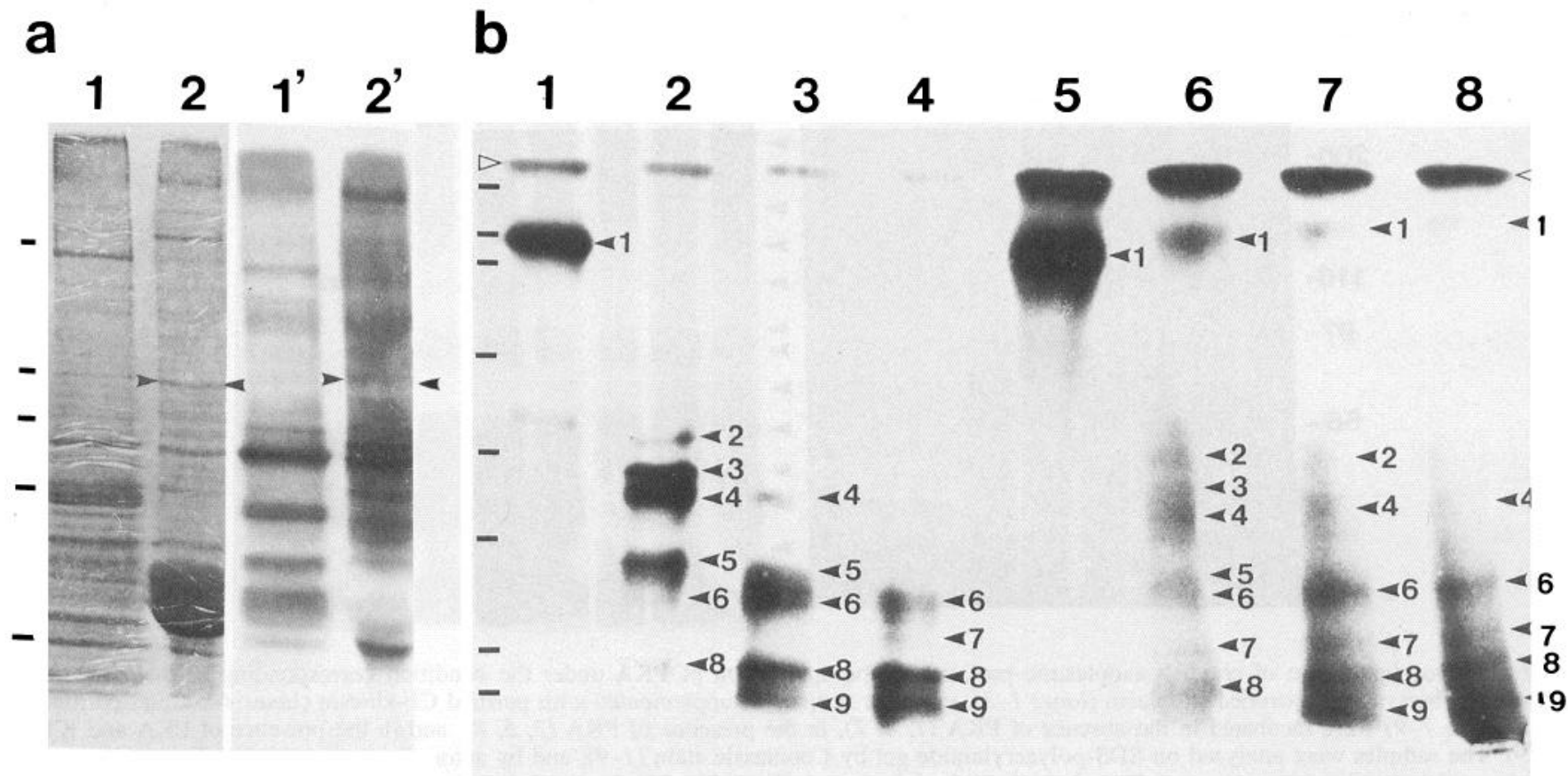

Figure 7. Kinesin phosphorylation in vivo. a, Metabolically labeled CF-kinesin was partially purified by cosedimentation with microtubules. Lanes 1 and 2 are Coomassie stains of crude extract and microtubule pellet, respectively. Lanes $1^{\prime}$ and $2^{\prime}$ are the corresponding autoradiogram. Arrowheads show the position of CF-kinesin band. $b$, Phosphopeptide mapping of PKA phosphorylated CF-kinesin (lanes $1-4)$ and in vivo phosphorylated CFkinesin (lanes 5-8). Phosphorylated CF-kinesin band was excised from SDS gels and digested with 0 (lanes 1 and 5 ), 3 (lanes 2 and 6 ), 10 (lanes 3 and 7), or 30 ng (lanes 4 and 8 ) of V8 protease. Peptides were separated on a second 15\% SDS gel (Cleveland et al., 1977), and exposed for autoradiography. Nine phosphopeptide bands were identified (arrowheads) in both PKA phosphorylated (lanes 1-4) and in vivo phosphorylated (lanes 5-8) preparations, suggesting that the in vivo phosphorylation site(s) is the same as the in vitro PKA phosphorylation site(s). Open arrowhead shows the position of the border between the stacking and the separation gels. The positions of molecular weight markers are indicated on the left of lane 1: $200,116,97,66,45,31,21$, and $14 \mathrm{kDa}$ from the top.

served change in the binding affinity was a little bit smaller, probably due to the impurity of the organelle fraction.

\section{Discussion}

Many types of regulatory machinery are postulated in the fast axonal transport, but only a few studies have shed any light on it. Phosphorylation/dephosphorylation is supposed to play a role in the regulation of fast axonal transport from the analogy of the regulation of the pigment granule movement in fish scale chromatophores (Lynch et al., 1986a,b; Rodzial and Haimo, 1986a,b; Sammak et al., 1992), but no positive evidence was reported yet. Bloom and his colleagues (1993) have reported the possible regulatory roles of small GTP-binding proteins in the squid giant axon, but they could not detect any effect by the inhibition of the kinase/phosphatase system. In this paper, we first report that the activation of PKA pathway selectively inhibits the anterograde vesicle transport by using crayfish giant axon as the experimental model.

\section{Activation of PKA pathway selectively inhibits anterograde vesicle transport}

The application of dbcAMP or exogenous PKA caused a transient but selective inhibition of the anterograde vesicle transport. This inhibition was completely reversed by the application of KT5720, and was augmented by ATP $\gamma$ S. Thus it is natural to assume that this inhibition is caused by the activation of PKA, but then why did phosphatase inhibitors and PKA inhibitors alone have no significant effect on organelle transport? We consider that it is due to the following reasons.
First, the phosphatase inhibitors that Bloom et al. (1993) and we used are okadaic acid or calyculin A. They inhibit only protein phosphatases $1,2 \mathrm{~A}$, and $2 \mathrm{~B}$, and are not effective to other phosphatases. This is why we used ATP $\gamma \mathrm{S}$ to inhibit all the putative phosphatases, and its application indeed augmented the PKA action. However, we could not detect any significant specific effects by ATP $\gamma \mathrm{S}$ alone. This would be due to the permeabilization and the dose of ATP $\gamma \mathrm{S}$. By permeabilization, soluble proteins including kinase were washed out. The dose of ATP $\gamma \mathrm{S}$ was arranged to be as low as possible to avoid its direct inhibitory effect on motor protein; it was arranged to have no apparent effect in the control experiments.

Second, Bloom et al. (1993) and we observed that the application of PKA inhibitor alone caused no increase in the anterograde vesicle transport. This might be partly due to the very low basal PKA activity in the axon; PKA might be already inhibited by the regulatory mechanism. However, we consider that the chief reason lies in the intrinsic limit of video light microscopy.

The resolution of light microscopy is limited. Therefore, when too many organelles are moving in the same direction, they will create a group of moving overlapped Airy disks. Because it is difficult to correctly estimate the number of organelles forming each Airy disks, only the number of Airy disks was counted instead of that of organelles. Thus, the number counted in this assay might be an underestimated value, especially when too many organelles are moving. In fact, judging from the apparent optical density of the Airy disks in our system, we consider that this overlapping effect was obvious when the number of moving vesicles exceeds 200. This intrinsic limitation of video micros- 


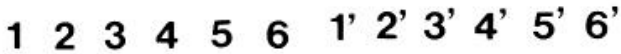
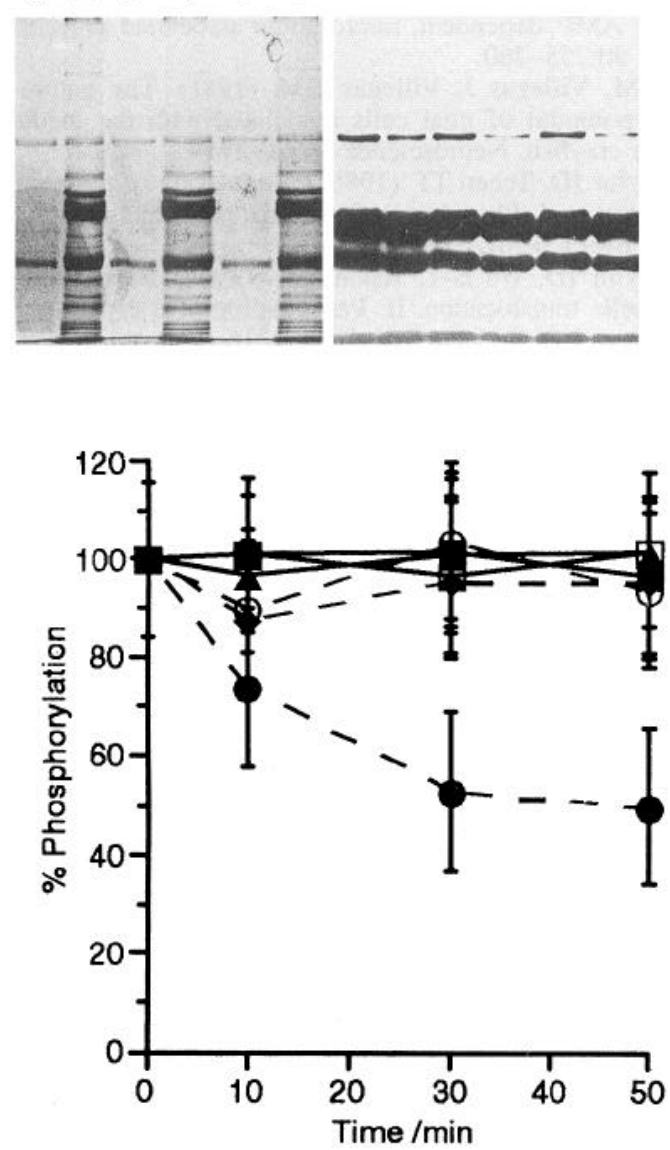

Figure 8. Phosphatase activity in the crayfish axoplasm. Purified CFkinesin was phosphorylated as in lanes 8 and $8^{\prime}$ in Figure 6 . After the addition of excess cold ATP, okadaic acid, calyculin A, and protease inhibitors, the sample was then incubated in the absence $(1,3,5)$ or in the presence $(2,4,6)$ of the extruded axoplasm for 10 min $(1,2), 30$ $\min (3,4)$, and $50 \mathrm{~min}(5,6)$. They were analyzed on SDS-polyacrylamide gel by Coomassie stain $(1-6)$ and by autoradiogram $\left(I^{\prime}-6^{\prime}\right)$. The position of CF-kinesin and two other contamination bands (p70 and tubulin) are indicated. The autoradiogram was quantitatively analyzed and the relative level of phosphorylation was plotted. Circle, square, and triangle symbols indicate kinesin, p70, and tubulin, respectively. The closed symbols with dotted line and the open symbols with solid line are in the presence and the absence of axoplasm, respectively. Mean and SEM of three independent experiments are shown. CF-kinesin was selectively and significantly dephosphorylated in the presence of axoplasm $(p<0.01$ at 30 and 50 min by paired $t$ test).

copy can limit the detection of the increase or the subtle decrease in the anterograde vesicle transport, for the number of moving vesicles in unperturbed axons was around this limit.

\section{Phosphorylation regulates anterograde vesicle transport}

What causes the selective inhibition of anterograde motility by PKA?

It seems unlikely that this inhibition is caused by nonphysiological hyperphosphorylation of axonal proteins. PKA activation can cause metabolic effects, but the selective inhibition could not be reproduced by altering ATP concentration. PKA can also phosphorylate cytoskeletal proteins, which perturbs axonal transport in Aplysia (Forscher et al., 1987) and in mammalian neuron (Sato-Yoshitake, unpublished results). In our system, no apparent change was observed in the cytoskeletal

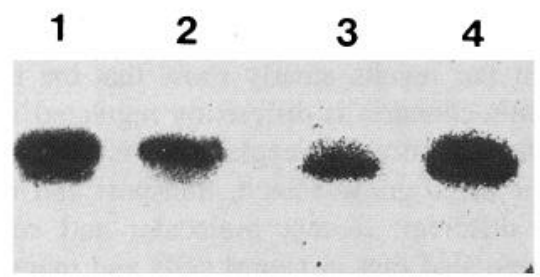

Figure 9. The binding of CF-kinesin to axonal organelles was weakened by the phosphorylation of CF-kinesin. Purified CF-kinesin and purified axonal organelles were incubated, and free kinesin and bound kinesin were separated by centrifugation. The amount of free $(3,4)$ and bound $(1,2)$ kinesin was quantitated by immunoblotting with anti-kinesin heavy chain monoclonal antibody $\mathrm{H} 2$ (Pfister et al., 1989). Lanes $I$ and 3 show the result using unphosphorylated kinesin, and lanes 2 and 4 show that with phosphorylated kinesin. The amount of kinesin bound to the organelles was reduced to $75 \pm 10 \%$ (mean \pm SEM, $N$ $=5, P<0.01$ by paired $t$ test) by phosphorylation.

architecture even at an electron microscopic level. Considering that only anterograde vesicle transport was selectively inhibited, it is natural to assume that the target of PKA phosphorylation is some proteins specifically involved in anterograde vesicle transport. Thus it is unlikely that cytoskeletal proteins are the PKA target responsible for the inhibition.

Therefore, the PKA target would be a protein whose phosphorylation selectively inhibits anterograde vesicle transport. That is, the anterograde vesicle transport can be regulated through the phosphorylation state of this PKA target protein. This is the first positive result supporting the idea that axonal transport is regulated through phosphorylation as in other cellular processes including pigment granule movement.

For further analysis, it is necessary to identify the PKA target. It will be among the proteins identified in Figure 6, and we have demonstrated that one of them is kinesin, which is supposed to be regulated by PKA phosphorylation (Sato-Yoshitake et al., 1992). Kinesin is a phosphoprotein in vivo whose phosphorylation was augmented by PKA activation. The site of phosphorylation in vivo is the same or similar to the PKA phosphorylation site in vitro, and the phosphorylation reduced the affinity to its cargo. These results are consistent with our previous in vitro results with rodent kinesin (Sato-Yoshitake et al., 1992), and they strongly support our hypothesis on the regulation of the direction of axonal transport that phosphorylation regulates the decommission of kinesin at the axon terminal by releasing it from its cargo (Sato-Yoshitake et al., 1992). Therefore, kinesin is a good candidate for the PKA target responsible for the regulation of axonal transport, although we could not demonstrate the direct relation between kinesin phosphorylation and the inhibition of anterograde vesicle transport. Furthermore, this does not mean that other putative PKA target proteins are not involved in the regulation. Further study will be necessary for their characterization and the analysis on the relation between their phosphorylation and the inhibition of vesicle transport.

\section{Different response of vesicles and mitochondria to PKA}

The movement of mitochondria is apparently different from that of vesicles. Vesicles move very smoothly and rarely change direction (Breuer et al., 1975), while the movement of mitochondria is very saccadic. They move for relatively short distance and then stop for a while. Their reversal of direction is not a rare event (Hirokawa and Yorifuji, 1986; Forman et al., 1987). It is not unnatural to suppose that the direction of movement is 
differently regulated. Only a few studies have focused on this difference, but our results clearly show that the movement of vesicles and mitochondria is differently regulated.

One possible and attractive explanation is that this is because the motors for anterograde vesicle transport and mitochondria transport are different. Recent molecular and cell biological studies have revealed that in neural cells and many other types of cells there exist new motor molecules besides kinesin and dynein, and it is supposed that these motors carry many different cargoes (Stewart et al., 1991; Aizawa et al., 1992; Kondo et al., 1994; Sekine et al., 1994). In Reticulomyxa, for example, it is reported that the bidirectional movement of mitochondria, even its plus-end-directed movement, is supported by a variant of cytoplasmic dynein (Euteneuer et al., 1988). Furthermore, we have recently identified a new motor protein, KIF1B, which is a good candidate for mitochondrial transport (Nangaku et al., 1994). The differential regulation of vesicle and mitochondria transports will be an important question for the future challenge.

\section{References}

Aizawa H, Sekine Y, Takemura R, Zhang Z, Nangaku M, Hirokawa N (1992) Kinesin family in murine central nervous system. J Cell Biol 119:1287-1296.

Bloom GS, Richards BW, Leopold PL, Ritchey DM, Brady ST (1993) GTP $\gamma \mathrm{S}$ inhibits organelle transport along axonal microtubules. J Cell Biol 120:467-476.

Breuer AC, Christian CN, Henkart M, Nelson PG (1975) Computer analysis of organelle translocation in primary neuronal cultures and continuous cell lines. J Cell Biol 65:562-576.

Burton PR, Hinkley RF, (1974) Further electron microscopic characterization of axoplasmic microtubules of the ventral nerve cord in crayfish. J Submicrosc Cytol 6:311-326.

Cleveland DW, Fisher SG, Kirschner MW, Laemmli UK (1977) Peptide mapping by limited proteolysis in sodium dodecyl sulfate and analysis by gel electrophoresis. J Biol Chem 252:1102-1106.

Dunkley PR, Jarvie PE, Rostas JAP (1988) Distributions of calmodulin- and cyclic AMP stimulated protein kinases in synaptosomes. $\mathrm{J}$ Neurochem 51:57-68.

Euteneuer U, Koonce MP, Ptister KK, Schliwa M (1988) An AlPase with properties expected for the organelle motor of giant amoeba, Reticulomyxa. Nature 332:176-178.

Forman DS, Lynch KJ, Smith RS (1987) Organelle dynamics in lobster axons: anterograde, retrograde, and stationary mitochondria. Brain Res 412:96-106.

Forscher P, Kaczmarek LK, Buchanan J, Smith SJ (1987) Cyclic AMP induces changes in distribution and transport of organelles within growth cones of Aplysia bag cell neurons. J Neurosci 7:3600-3611.

Hirokawa N (1986) $270 \mathrm{kD}$ microtubule-associated protein cross-reacting with anti MAP2 IgG in the crayfish peripheral nerve axon. J Cell Biol 103:33-39.

Hirokawa N, Yorifuji H (1986) Cytoskeletal architecture of reactivated crayfish axons, with special reference to crossbridges among microtubules and between microtubules and membrane organelles. Cell Motil Cytoskel 6:458-468.

Hirokawa N, Yoshida Y, Sato-Yoshitake R, Kawashima T (1990) Brain dynein localizes on both anterogradely and retrogradely transported membranous organelles. J Cell Biol 111:1027-1037.

Hirokawa N, Sato-Yoshitake R, Kohayashi N, Pfister KK, Bloom GS, Brady ST (1991) Kinesin associates with anterogradely transported membranous organelles in vivo. J Cell Biol 114:295-302.

Hollenbeck PJ (1993) Phosphorylation of neuronal kinesin heavy and light chains in vivo. J Neurochem 60:2265-2275.

Kase H, Iwahashi K, Nakanishi S, Matsuda Y, Yamada K, Takahashi M, Murakata C, Sato A, Kanleko M (1987) K-252 compounds, novel and potent inhibitors of protein kinase $\mathrm{C}$ and cyclic nucleotide-dependent protein kinases. Biochem Biophys Res Commun 142:436440.

Kondo S, Sato-Yoshitake R, Noda Y, Aizawa H, Nakata T, Matsuura Y, Hirokawa N (1994) KIF3A is a new microtubules based fast anterograde motor in the nerve axons. J Cell Biol 125:1095-1107.
Leterrier J-F, Liem RKH, Shelanski ML (1981) Preferential phosphorylation of the 150,000 molecular weight component of neurofilaments by a cyclic AMP-dependent, microtubule-associated protein kinase. J Cell Riol 90:755-760.

Lieberman EM, Villegas J, Villegas GM (1981) The nature of the membrane potential of glial cells associated with the medial giant axon of the crayfish. Neuroscience 6:261-271.

Lynch TJ, Taylor JD, Tchen TT (1986a) Regulation of pigment organelle translocation. I. Phosphorylation of the organelle-associated protein p57. J Biol Chem 261:4204-4211.

Lynch TJ, Taylor JD, Wu B-Y, Tchen TT (1986b) Regulation of pigment organelle translocation. II. Participation of a cAMP-dependent protein kinase. J Biol Chem 261:4212-4216.

Matthies HJG, Miller RJ, Palfrey HC (1993) Calmodulin binding to and cAMP-dependent phosphorylation of kinesin light chains modulate kinesin ATPase activity. J Biol Chem 268:11176-11187.

Murphy DB, McNiven MA, Wallis KT, Kuznetsov SA, Gelfand VI (1989) The phosphorylation of kinesin does not affect its ATPase and translocating activities. J Cell Biol 109:80a.

Nairn AC, Hemmings HC Jr, Greengard P (1985) Protein kinases in the brain. Annu Rev Biochem 51:931-976.

Nangaku M, Sato-Yoshitake R, Okada Y, Noda Y, Takemura R, Yamazaki H, Hirokawa N (1994) KIF1B: a new microtubule plus-end directed monomeric motor for transport of mitochondria. Cell, in press.

Nestler EJ, Greengard P (1989) Protein phosphorylation and the regulation of neuronal function. In: Basic neurochemistry: molecular, cellular, medical aspects (Siegel GS, ed), pp 373-398. New York: Raven.

Pfister KK, Wagner MC, Stenoien DL, Brady ST, Bloom GS (1989) Monoclonal antibody to kinesin heavy and light chains stain vesiclelike structures, but not microtubules, in cultured cells. J Cell Biol 108:1453-1463.

Rodzial MM, Haimo LT (1986a) Reactivated melanophore motility: differential regulation and nucleotide requirements of bidirectional pigment granule transport. J Cell Biol 103:2755-2764.

Rodzial MM, Haimo LT (1986b) Bidirectional pigment granule moveIntents of melanophores are regulated by protein phosphorylation and dephosphorylation. Cell 47:1061-1070.

Sammak PJ, Adams SR, Harootunian AT, Schliwa M, Tsien RY (1992) Intracellular cyclic AMP, not calcium, determines the direction of vesicle movement in melanophores: direct measurement by fluorescence ratio imaging. J Cell Biol 117:57-72.

Sato-Yoshitake R, Yorifuji H, Inagaki M, Hirokawa N (1992) The phosphorylation of kinesin regulates its binding to synaptic vesicles. J Biol Chem 267:23930-23936.

Sekine Y, Okada Y, Noda Y, Kondo S, Aizawa H, Takemura R, Hirokawa N (1994) A novel microtubule-based motor protein (KIF4) for organelle transports, whose expression is regulated developmentally. J Cell Biol 127:187-201.

Shimizu T, Furusawa K, Ohashi S, Toyoshima YY, Okuno M, Malik F, Vale RD (1991) Nucleotide specificity of the enzymatic and motile activities of dynein, kinesin, heavy meromyosin. J Cell Biol 112: 1189-1197.

Smith RS (1980) The short term accumulation of axonally transported organelles in the region of localized lesions of single myelinated axons. J Neurocytol 9:39-65.

Stewart RJ, Pesavento PA, Woerpel DN, Goldstein LSB (1991) Identification and partial characterization of six members of the kinesin superfamily in Drosophila. Proc Natl Acad Sci USA 88:8470-8474.

Vale RD, Reese TS, Sheetz MP (1985a) Identification of a novel forcegenerating protein, kinesin, involved in microtubule-based motility. Cell 42:39-50.

Vale RD, Schnapp BJ, Mitchison T, Steuer E, Reese TS, Sheetz MP (1985b) Different axoplasmic proteins generate movement in oppositc dircctions along microtubules in vitro. Cell 43:623 632.

Viancour TA, Seshan KR, Bittner GD, Sheller RA (1987) Organization of axoplasm in crayfish giant axons. J Neurocytol 16:557-566.

Wagner MC, Pfister KK, Bloon GS, Brady ST (1989) Copurification of kinesin polypeptides with microtubule-stimulated Mg-ATPase activity and kinetic analysis of enzymatic properties. Cell Motil Cytoskel 12:195-215.

Walter U, Kanof P, Schulman H, Greengard P (1978) Adenosine 3',5'monophosphate receptor proteins in mammalian brain. J Biol Chem 253:6275-6280. 